\title{
Probe beam image detection for mode-mismatched dual beam thermal lens spectrometry
}

\author{
J. Shen, A.J. Soroka, M.L. Baesso and R.D. Snook \\ DIAS, UMIST, P.O. Box 88, Manchester, M60 IQD, U.K.
}

\begin{abstract}
A$ model for $C W$ induced mode-mismatched dual-beam thermal lens spectrometry using the whole probe beam profile has been developed. This model quantitatively describes the intensity distribution before and after the thermal lens has been created. It is considered that after passing through the thermal lens, the TM ${ }_{00}$ Gaussian probe beam becomes the superposition of several Gaussian beams of different parameters. A compact mathematical expression was obtained, which allows absolute measurements of opthothermal properties of transparent samples. Experimental proofs have been displayed.
\end{abstract}

\section{INTRODUCTION}

Noise affects the sensitivity and reproducibility of TLS. Noise can be time-dependent, or spatial noise [1]. Since the spot size change of the probe beam at the detector plane is generally inferred from the variation of the intensity at the centre of the probe beam at the detector plane, and is sensitive to the relative positions of the probe beam centre and pinhole-photo-sensor assembly, any perturbation disturbing the Gaussian profile of the beams can lead to noise and errors in the thermal lens measurements. The spatial noise, which causes problems in the centre intensity measurement technique, might be less troublesome in the scheme that monitors the intensity profile of the entire probe beam. In the modemismatched dual-beam thermal lens experiment, the well defined Gaussian profile of the probe beam is destroyed, due to the possible size mismatching between the dimensions of the excitation and probe beam in the sample. It is, therefore, necessary to have a theoretical model for the mode-mismatched dual-beam TLS to carry out the beam profile analysis by curve fitting to a known mathematical expression to average the spatial noise.

\section{II.EXPERIMENTAL}

The experimental procedure has been described elsewhere[2,3]. Here, the setup is shown in figure 1. An one dimensional photodiode array (Reticon K Series, RL1024), which has 1024 elements with a separation of $25 \mu \mathrm{m}$ between two element centres, was used to detect the intensity profile of the probe beam. This was used in conjunction with an R100B (Reticon) mother board to provide video output and start pulse signals and a home made data acquisition board connected to 486 computer. The data acquisition board operated at a sampling frequency of $853 \mathrm{KHz}$, thus allowing each point captured to represent an element in the photodiode array. The sample used was a $0.0001 \mathrm{M}$ aqueous solution of $\left(\mathrm{CuSO}_{4}\right) \cdot 5 \mathrm{H}_{2} \mathrm{O}$ and Ethylenediaminetetraacetic acid, disodium salt, dihydrate (EDTA). 


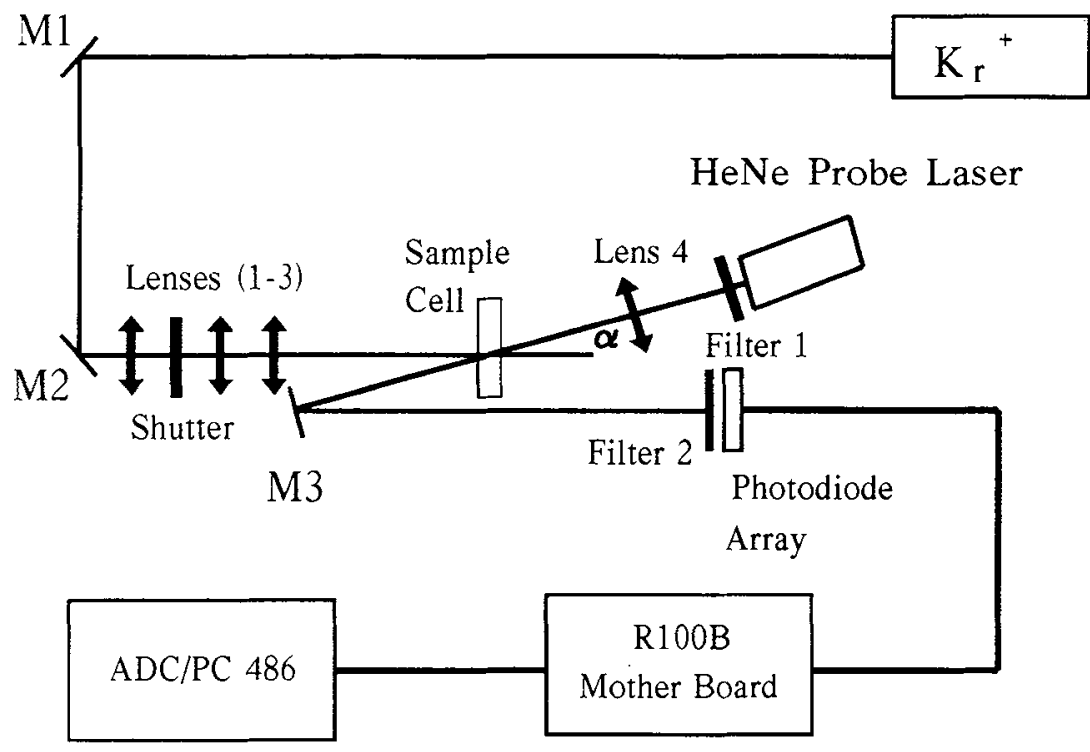

Figure 1. A schematic diagram of experimental apparatus.

\section{RESULTS AND DISCUSSION}

Figure 2 shows the normalized steady-state probe beam intensity distribution, in a parabolic thermal lens model [4] and in the aberrant model at the detector plane in the far field. In the mode-matched dualbeam or single beam thermal lens experiment, the probe beam intensity distributions predicted by the parabolic model and aberrant model are close when $\theta$ is small. In the mode mismatched dual-beam thermal lens experiment, there is a considerable difference between the predictions of the models, even for small $\theta$ (figure $2 \mathrm{~b}$ ). Furthermore, the interference fringes are not predicted by the parabolic treatment because the thermal lens is treated as an ideal thin lens.
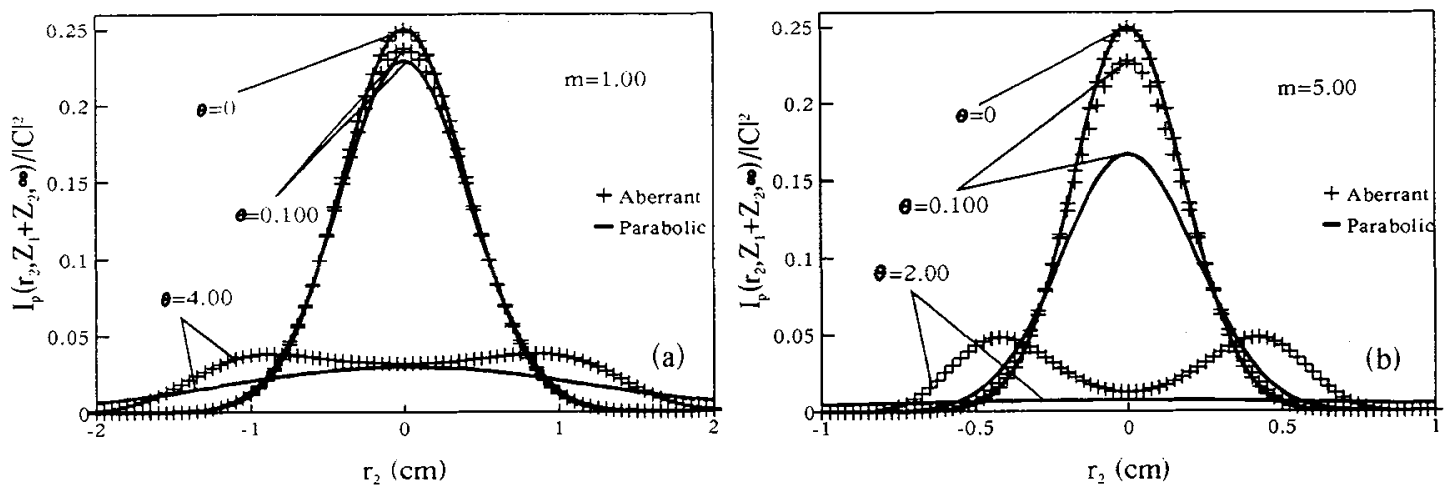

Figure 2. Theoretical comparisons of the normalized probe beam intensity distributions in the parabolic and aberrant models at the detector plane: (a) $m=1.00,(b) m=5.00, . Z_{2}=100 \mathrm{~cm}, \mathrm{v}=3.00^{1 / 2}$ 
An approximation for the aberrant model has been made to obtain a compact analytical expression, which can be used to process experimental data. According with this treatment, after passing through the thermal lens, the probe beam can be though as the superposition of several Gaussian beams of different parameters, and the probe beam intensity distribution at the detector plane is then the interference pattern of the Gaussian beams. In this approximation, the intensity distribution of the probe beam at the detector plane can be expressed as $[4]$ :

$$
\begin{gathered}
I\left(I_{2}, Z_{1}+Z_{2}, \infty\right) \approx|C|^{2} H_{0}^{2} \exp \left(-2 F_{0} Y^{2} r_{2}^{2}\right) \\
+|C|^{2} \theta H_{0} \sum_{n=1}^{4} H_{n} \exp \left[-\left(F_{n}+F_{0}\right) Y^{2} I_{2}^{2}\right] \sin \left[\left(\boldsymbol{\beta}_{n}-\beta_{0}\right) Y^{2} I_{2}^{2}+\left(\boldsymbol{\alpha}_{n}-\boldsymbol{\alpha}_{0}\right)\right] \\
F_{n}=\frac{1-b_{n} m}{\left.4\left[\left(1-b_{n} m\right)^{2}+V^{2}\right)\right]}, \quad H_{n}=\frac{a_{n}}{\sqrt{\left.\left(1-b_{n} m\right)^{2}+V^{2}\right)}} \\
\boldsymbol{\alpha}_{n}=\tan ^{-} 1\left(-\frac{V}{1-b_{n} m}\right), \quad \boldsymbol{\beta}_{n}=\frac{V}{4\left[\left(1-b_{n} m\right)^{2}+V^{2}\right]} \\
\theta=-\frac{p_{\theta} A_{\theta} I}{k \lambda_{p}} \frac{d s}{d T}, \quad Y=\frac{2 \pi}{\lambda_{p}} \frac{\omega_{\theta}}{Z_{2}} \sqrt{m} \\
m=\left(\frac{\omega_{1 p}}{\omega_{\theta}}\right)^{2}, \quad V=\frac{Z_{1}}{Z_{c}}+\frac{Z_{c}}{Z_{2}}\left[1+\left(\frac{Z_{1}}{Z_{c}}\right)^{2}\right]
\end{gathered}
$$

where, $\theta$ is approximately the phase difference of the probe beam between the beam centre and $r_{1}=\sqrt{ }\left(2 \omega_{\mathrm{e}}\right)$; $\omega_{1 p}$ is the radius of the probe beam in the sample; $\omega_{\mathrm{e}}$ is the radius of the excitation beam in the sample; $\mathrm{P}_{\mathrm{e}}$ is the excitation laser beam power; $\mathrm{A}$ is the sample optical absorption coefficient at the excitation laser beam wavelength; 1 is the sample thickness; $\lambda_{p}$ is the probe beam wavelength; ds/dT is the temperature coefficient of the optical path length; $K$ is the sample thermal conductivity and $a_{n}$ and $b_{n}$ can be determined by numerical calculation [4]; $Z_{1}$ is the distance between the probe beam waist and the sample; $Z_{2}$ is the distance between tha sample and the detector; $Z_{c}$ is the confocal distance of the probe beam.

$\begin{array}{ccc}\mathrm{n} & \mathrm{a}_{\mathrm{n}} & \mathrm{b}_{\mathrm{n}} \\ 0 & 1 & 0 \\ 1 & 1.440 & -1.027 \\ 2 & -2.047 & -0.03816 \\ 3 & -1.596 & -0.2136 \\ 4 & 5.083 & 0.001531\end{array}$

For the curve fitting with the experimental data shown in figure $3 \mathrm{a},|\mathrm{C}|^{2}$ was obtained by fitting the recorded intensity profile of the probe beam before the thermal lens is established and by using only the first term in equation 1 . The intensity profile of the probe beam recorded after the thermal lens was then fitted to the whole equation to find $\theta$. The value was $\theta=0.100 \pm 0.003$, when the expected value is $\theta_{e}=0.0984$, which is less than $2 \%$ of difference. However, when the parabolic treatment was used, $\theta$ $=0.0206$ (figure $3 \mathrm{~b}$ ). 

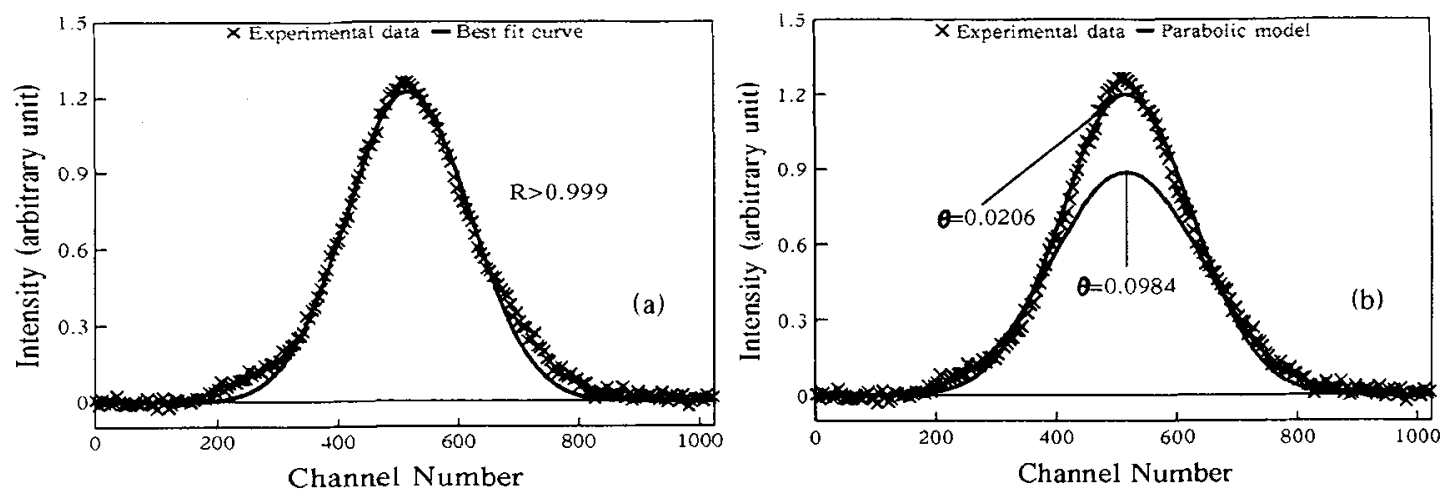

Figure 3. Experimental data of the He-Ne laser as the probe beam and their best fit curves: (a) fitting to the aberrant model, (b) fitting to the parabolic model.

In conclusion, this work presents a clear physical picture of the thermal lens effect. The sample placed in the $\mathrm{TEM}_{00}$ Gaussian excitation beam is heated by the absorbed laser energy, producing an optical path length variation, which causes an additional phase shift on the $\mathrm{TEM}_{00}$ Gaussian probe beam and distorts its wave front. The probe beam emerging from the sample is no longer a Gaussian beam, and becomes a superposition of several Gaussian beams of differences parameters. The whole beam profile at the detector plane is then the interference pattern of these Gaussian beams. Besides, this model provides a compact mathematical expression, with which optothermal properties of the transparent samples can be absolutely measured. Moreover, this work presents strong evidence that for the mode-mismatched thermal lens configuration, the parabolic model cannot be applied for quantitative measurement.

\section{REFERENCES}

1. Jasen, K.L. and Harris, J.M., Anal. Chem., 57, 1698 (1985)

2. Baesso, M.L., Shen, J. and Snook, R.D., J. Appl. Phys., (In Press), (1994)

3. Shen, J., Baesso, M.L. and Snook, R.D., J. Appl. Phys., (In Press), (1994)

4. Shen, J., "Theoretical Modelling of Photothermal Lens Spectrometry and Its Experimental Applications",

Ph.D. Thesis, UMIST, Manchetster, U.K. (1993) 